\title{
Structural rejuvenation in bulk metallic glasses
}

\author{
Y. Tong ${ }^{1}$, T. Iwashita ${ }^{2}$, W. Dmowski ${ }^{1 *}$ H. Bei ${ }^{3}$, Y. Yokoyama $^{4}$, and T. Egami ${ }^{1,2,3}$ \\ ${ }^{1}$ Department of Materials Science and Engineering, University of Tennessee, Knoxville, TN 37996 \\ ${ }^{2}$ Department of Physics and Astronomy, University of Tennessee, Knoxville, TN 37996 \\ ${ }^{3}$ Oak Ridge National Laboratory, Oak Ridge, TN 37831 \\ ${ }^{4}$ Institute for Materials Research, Tohoku University, Katahira, Sendai 980-8577, Japan
}

\begin{abstract}
Using high energy X-ray diffraction we study structural changes in bulk metallic glasses after uniaxial compressive homogeneous deformation at temperatures slightly below the glass transition. We observe that deformation results in structural disordering corresponding to an increase in the fictive, or effective, temperature. However, the structural disordering saturates after yielding. Examination of the experimental structure and molecular dynamics simulation suggests that local changes in the atomic connectivity network are main driving force of the structural rejuvenation.
\end{abstract}

Keywords: Bulk metallic glasses, structure, rejuvenation, creep, X-ray diffraction. 


\section{Introduction.}

When bulk metallic glasses (BMGs) are subjected to stress at room temperature most of them fail catastrophically through the formation and slip of shear bands without much plasticity $[1,2]$. Only a small number of glass systems, mostly based on noble metals with a high Poisson ratio, exhibit room temperature plasticity [3-6]. But even ductile BMGs are sensitive to annealing-induced embrittlement [7-10]. Therefore, effective and simple methods to improve room temperature plasticity are sought after, and numerous methods have been explored. Generally, they can be categorized into homogeneous [8, 11] and inhomogeneous types [12-15]. In the homogeneous method structural rejuvenation occurs in the whole volume, whereas the inhomogeneous method relies upon localized plastic deformation resulting in multiple shear bands introduced into BMGs. The homogeneous method is more attractive since it eliminates the risk of damaging BMG samples by stressing them beyond the yield point. Recently, an effective homogeneous method to recover plasticity by using thermo-mechanical creep has been found [8]. A structural study of the BMGs samples that showed creep-induced plasticity indicated signature of microscopic structural rejuvenation [8]. However, the atomistic mechanism of structural rejuvenation remains elusive. Also it remains to be determined which part of the creep (anelastic-recoverable or plastic) is contributing to rejuvenation. The purpose of this work is to determine the atomistic mechanism of rejuvenation through the experimental structural study using high-energy x-ray diffraction and computer simulation.

The glassy state is characterized by a fictive, or effective, temperature $\left(T_{\mathrm{f}}\right)$ corresponding to the supercooled liquid state into which the glass is frozen $[16,17]$. 
Accordingly, physical implication of structural rejuvenation is the increase in $T_{\mathrm{f}}$. The numerical simulation based upon the shear transformation zone (STZ) concept [16] suggests that $T_{\mathrm{f}}$ is a function of a strain rate, and so deformation at a sufficiently high strain-rate could generate structural rejuvenation. From both theoretical and practical perspective, it is important to validate these predictions. In this paper, we examine experimentally structural rejuvenation in relation to the strain rate and deformation stage. Because direct measurement of $T_{\mathrm{f}}$ is impractical we carried out structural studies of BMGs deformed at different strain rate assuming that a particular $T_{\mathrm{f}}$ corresponds to a unique structural state of a glass characterized by a PDF. The fictive temperature can be related to the pressure fluctuations in the glassy structure [e.g. 18] which can be related to the PDF peak width and thus peak height. It was shown that $\left\langle p^{2}\right\rangle$ (where $p$ is atomic level pressure) is related to the height and width of the RDF peak $[19,20]$. The distribution of $\left\langle p^{2}\right\rangle$ reflects the structural disorder in glass. For example, if the sample is annealed and undergoes structural relaxation, the distribution becomes narrow, the peak width becomes narrower and the peak height becomes higher. The opposite is true if we introduce disordering. In this study we examined the change in the averaged PDF peak height which linearly tracks the fictive temperature. In principle the fictive temperature can be directly determined by knowing the PDF of the samples equilibrated at various temperatures. In this study we did not carry out such an elaborate calibration procedure, so that the $T_{f}$ is only semi-quantitatively known from the PDF. Then, combining experimental results with a qualitative simulation study of the structural evolution in a model glass during fixed strain-rate deformation we elucidate the structural micro- 
mechanism of rejuvenation. We demonstrate that the atomistic mechanism of structural rejuvenation is due to topological re-arrangement in the atomic connectivity network.

\section{Experiment}

BMG having composition $\mathrm{Zr}_{55} \mathrm{Cu}_{30} \mathrm{Ni}_{5} \mathrm{Al}_{10}\left(T_{g}=434{ }^{\circ} \mathrm{C}\right)$ was prepared by a tilt casting method [21]. Slabs with dimensions of $2.5 \mathrm{~mm} \times 2.5 \mathrm{~mm} \times 5 \mathrm{~mm}$ were cut from a single as-cast rod using electric discharge machining (EDM). All samples were annealed at $400{ }^{\circ} \mathrm{C}$ for $24 \mathrm{hrs}$ to acquire the same reference state. Then they were polished to remove contamination on their surfaces. Using a uniaxial mechanical testing system (Instron 5881, Shakopee, MN), displacement controlled compression tests were conducted at the $400{ }^{\circ} \mathrm{C}$, which is the same as annealing temperature but lower than glass transition temperature. Samples were first heated and stabilized at $400{ }^{\circ} \mathrm{C}$ for $20 \mathrm{~min}$ altogether. After the loading test, samples were immediately quenched into iced water. Plastic strain for each sample was measured using a caliper with an accuracy of $\pm 0.005 \mathrm{~mm}$.

A thin slice parallel to the loading axis was cut from each sample by EDM and then fine polished into pieces with $0.5 \mathrm{~mm}$ thickness for X-ray diffraction measurements. High energy X-ray study was carried out at the 6-ID and 1-ID beam lines of the Advanced Photon Source, Argonne National Laboratory. The beam energy was $100 \mathrm{keV}$. A two-dimensional (2-D) stationary detector, placed $\sim 34 \mathrm{~cm}$ behind the sample and having $2048 \times 2048$ pixels with $200 \times 200 \mu^{2}$ pixel size, was used to collect diffraction patterns. Calibration was performed using $\mathrm{CeO}_{2}$ NIST powder standard. FIT2D software was used to correct for a beam polarization, dark current and data binning. 
Molecular dynamics studies were performed on a model of amorphous iron [22] with 16,584 atoms. A monoatomic system rather than the alloy used in the experimental study was chosen to eliminate the effect of chemical composition. A realistic MD simulation of an experimentally studied glass is not practical because of the required model size and low strain rates that cannot be reproduced in simulation. To resolve qualitatively changes in the local topology (thus ignoring chemical ordering) we use monoatomic model system with high strain rates achievable by simulation. Initially, the structure was prepared by an instant quench from a liquid at $1500 \mathrm{~K}$ to a glass at $700 \mathrm{~K}$, followed by cooling process at $0.2 \mathrm{~K} / \mathrm{ps}$. After gradual cooling to room temperature, the system was relaxed for $2 \mathrm{~ns}$ under NTP ensemble with pressure set to zero and temperature at $300 \mathrm{~K}$. Initially, the positions of all atoms were displaced with a uniform tensile strain of $2 \%$ and the Poisson ratio of 0.33 . The deformed structure was relaxed at $300 \mathrm{~K}$ with the effective strain rate of $10 \%$ s. During the relaxation process the pressure perpendicular to the tensile direction was kept to be zero. The system stays glassy at this strain rate. The pair distribution function $(\mathrm{PDF})$ was evaluated for two groups of atoms: atoms that changed their local topology by displacing from the first neighbor shell and atoms that maintained their original environment.

\section{Results and Discussion}

\subsection{Structural characterization.}

Samples quenched to room temperature following fixed stain-rate deformation show evidence of structural anisotropy in the plane parallel to the loading axis while they remain isotropic in the plane normal to the loading direction. This is evidenced in the 2-D 
diffraction patterns. For each plane, we measured two orientations rotated by $90^{\circ}$ about the axis parallel to the X-ray beam. The diffraction rings for the plane parallel to the stress are distorted and have elliptical shape. Therefore, the difference in the intensities of these two images shows characteristic 2-fold pattern as displayed in Figure 1(a). On the other hand, for plane normal to the stress, the diffraction pattern is isotropic and the difference between $0^{\circ}$ and $90^{\circ}$ shows negligible intensity fluctuations, as shown in Figure 1(b). Since the structure of BMG after deformation is no longer isotropic a spherical harmonics expansion [23-25] is employed to separate isotropic and anisotropic components of the structure function $S(Q)$ and the PDF $g(r)$ :

$$
S(\vec{Q})=\sum_{l, m} S_{l}^{m}(Q) Y_{l}^{m}\left(\frac{\vec{Q}}{Q}\right), \quad g(\vec{r})=\sum_{l, m} g_{l}^{m}(r) Y_{l}^{m}\left(\frac{\vec{r}}{r}\right)
$$

Because of the uniaxial symmetry anisotropic terms are limited to the $(l=2, m=$ 0) elliptical component. Next, by Bessel transformations isotropic part of the pair distribution function PDF, $g_{0}^{0}(r)$, and anisotropic component, $g_{2}^{0}(r)$, are obtained [23, 25]. The isotropic part of the PDF describes the average structure of the glass and is intimately related to the fictive temperature. The $g_{2}^{0}(r)$ component characterizes anisotropy introduced by high temperature deformation. During the high temperature deformation the applied stress induces local structural rearrangements (relaxation). After quenching and removing external stress, these local rearrangements produce local stress fields that add up to a non-zero stress, which is balanced by a long range stress due to macroscopic deformation of the sample. This macroscopic deformation is the signature of anelastic deformation and represents a memory state of the high temperature creep 
deformation under constant stress. The structural anisotropy due to this effect has been observed by previous X-ray diffraction studies [23-28].

The strain anisotropy due to affine (uniform) deformation gives rise to the elliptical $(l=2, m=0)$ component of the anistropic PDF:

$$
g_{2, \text { affine }}^{0}(\mathrm{r})=\varepsilon \frac{2(1+v)}{3 \sqrt{5}} r \frac{d}{d r} g_{0}^{0}(\mathrm{r})
$$

here, $\varepsilon$ is a uniaxial strain and $v$ is the Poisson's ratio. The $g_{2}^{0}(r)$ for a sample that underwent anelastic deformation is similar to the affine PDF beyond about $9 \AA$. However, at a shorter range it deviates significantly from eq. (2) [21-26]. The difference between $g_{2, a f f i n e}^{0}(r)$ and the experimental $g_{2}^{0}(r)$ represents the extent of the local structural re-arrangements. The strain fitted over the range beyond $9 \AA$ represents the recoverable anelastic strain. In the STZ phenomenology, local rearrangements define the shear transformed volumes, whereas elastically deformed matrix provides the restoring force. The elastic strain of the matrix corresponds to the recoverable strain mentioned above that can be determined from the fit to $g_{2}^{0}(r)$ at long range (beyond $9 \AA$ ) using eq. 2.

During deformation at a constant strain-rate the total strain consists of three components: affine (elastic), anelastic, and plastic strain. The elastic deformation is reversible upon unloading so it does not change the structure; anelastic deformation generates local structural relaxation that results in anisotropy, and changes induced by a plastic strain may result in structural disordering. Since we perform ex-situ X-ray diffraction study on the quenched samples we observe the memory of the structural 
changes during a fixed strain-rate experiment induced by anelastic and plastic deformation.

The stress-strain curves for samples deformed at increasing strain rates are shown in Figure 2(a). From these plots the flow stress, $\sigma_{f}$, is obtained and the dependence of viscosity on the strain rate is evaluated from $\sigma_{f}=3 \eta \dot{\varepsilon}$ [29], as displayed in Figure 2(b). The plot indicates that deformation is in the non-Newtonian regime. The quenched structures of BMGs after reaching the steady state flow (as shown in Fig. 2(a)) were studied using atomic pair distribution function (PDF) as described above.

Fig. 3 presents $g_{0}^{0}(r)$ and $g_{2}^{0}(r)$ for samples deformed at different strain rates. We found that changes in $g_{0}^{0}(r)$ are small but statistically significant, as clearly seen in the difference plots in Fig. 3(a) the middle panel. It has been observed that structural relaxation, thus a decrease in the fictive temperature, results in sharpening of the PDF peaks $[8,30-32]$. Because the main PDF peak is composed of sub-peaks we calculate average height by integrating area of the peak and dividing by its base at $g(r)=1$. The decrease in the averaged PDF peak height seen in Fig 3(a) bottom panel is evident of gradual disordering. It shows increase in the fictive temperature with increasing strain rate up to $2.5^{*} 10^{-4} \mathrm{~s}^{-1}$. Thus deformation indeed results in structural rejuvenation [16], but its extent depends on strain rate. It is also seen that structural rejuvenation effect has maximum at the strain rate of $2.5^{*} 10^{-4} \mathrm{~s}^{-1}$, above which an opposing change appears (Fig 3(a) the bottom panel). This is distinct from a monotonic increase in the fictive temperature (structural rejuvenation) predicted by MD simulations. However, this may be experimental artifact related to the limiting rate in our experiment. Further mechanical testing, exceeding strain rate 5.0*10-4s-1, (not shown) resulted in inhomogeneous plastic 
deformation. Therefore, the observed drop in the fictive temperature at the $2.5^{*} 10^{-4} \mathrm{~s}^{-1}$ strain rate may be related to strain localization before the fracture.

Differences in the amplitude of $g_{2}^{0}(r)$ (Figure 3(b) top panel) are rather small, suggesting that structural anisotropy only weakly depends on the flow stress. Furthermore, we find that the anisotropic $g_{2}^{0}(r)$ for samples after deformation at a constant strain-rate is virtually the same as $g_{2}^{0}(r)$ induced by thermo-mechanical creep (constant stress experiment) except for a scale, as indicated in Figure 3(b), the bottom panel. This confirms that structural anisotropy frozen in specimens deformed at constant strain rate originates from anelasticity.

It is important to know which part of the visco-elastic/plastic deformation contributes most to the rejuvenation process. Therefore, we also characterized the structural evolution after the deformation process at one selected strain rate, which resulted in largest decrease in peak height in Figure 3(a) bottom panel. We prepared a series of samples deformed to different total strains at a fixed strain rate $\left(2.5^{*} 10^{-4} \mathrm{~s}^{-1}\right)$ and the same temperature $\left(400^{\circ} \mathrm{C}\right)$. The stress-strain curves are shown in Fig. 4(a). From Fig. 4(a), we can see a pronounced stress-overshoot before a steady state is reached. Stress overshoot contains valuable information about the dynamics of the non-steady state, which is discussed from a structural perspective below. First, $g_{2}^{0}(r)$ is quantitatively analyzed to estimate the long range strain induced by frozen anelasticity. Fig. 4(b) shows the long-range strain obtained by fitting the experimental $g_{2}^{0}(r)$ to the derivative of the isotropic PDF over the $r$ range between $8.9 \AA$ and $20 \AA$ using eq. 2 . At this elevated temperature of $400{ }^{\circ} \mathrm{C}$ a truly elastic behavior is almost non-existent, and significant anelastic deformation is observed well below the overshoot strain. Following this 
overshoot, a steady state with a constant anelastic strain is eventually achieved. The overshoot in $g_{2}^{0}(r)$ indicates that anelasticity is reduced after overshoot in a manner apparently consistent with the anelastic-to-visco-plastic transition (APT) proposed by the STZ theory [33, 34]. In the STZ picture, anelasticity is generated by a shear transformation in isolated STZs confined in an elastic matrix and visco-plasticity is created by the percolation of isolated STZs [34]. When percolation occurs, confinement from elastic matrix disappears and therefore energy stored in these STZs is suddenly relaxed. This can be verified simply by comparing the changes in anelastic and plastic strains. The plastic strain for each sample is plotted against the total strain in Fig. 4(c). A sudden increase in plastic strain accompanying stress overshoot is observed, emphasized by a blue dash-line circle in Fig. 4(c). This surge matches the sudden decline in the anelastic strain (determined from $g_{2}^{0}(r)$ ), confirming the AP transition.

We now evaluate how structural rejuvenation proceeds with deformation in terms of the changes in $g_{0}^{0}(r)$. The changes in $g_{0}^{0}(r)$ are subtle, but definite variation is observed in the averaged PDF peak height in $g_{0}^{0}(r)$ (Fig. 4(d)). The decreasing peak height at the initial stages of the deformation process up to $7 \%$ indicates structural rejuvenation. Then the peak height, thus the corresponding fictive temperature, becomes stabilized when the anealstic strain drops after the overshoot. The comparison of the results in Fig 4(b) and 4(d) suggests that structural rejuvenation is caused by anelasticityrelated topological rearrangement. It is interesting to note that we could expect an undershoot behavior in the peak height change of $g_{0}^{0}(r)$, however, there is no such evidence in plot Fig 4(b). The missing undershoot indicates that visco-plasticity does not impact structural rejuvenation. The presented data in Fig. 4 suggest that structural 
rejuvenation is mainly driven by anelastic effects and cannot be enhanced much beyond a stress drop in a steady state regime where apparently creation and destruction of the STZs remains balanced.

\subsection{Molecular dynamics simulation.}

The atomistic mechanism of anelasticity has been understood as a local topological rearrangement of atoms in terms of bond-cutting and formation (BCF) based on experimental data and MD simulation [25]; however, its relation to the structural rejuvenation is not evident. Because rejuvenation happens by a change in the topology of atomic connectivity (mechanical deformation) we ignore chemical effects and study a monoatomic system. Here, MD simulation was performed to verify whether one common topological rearrangement induces both structural rejuvenation and anelasticity. In such

a case, $g_{0}^{0}(r)$ calculated for the atoms involved in the BCF rearrangements should show a structural rejuvenation effect. Fig. 5(a) compares the PDFs before and after deformation, for the atoms without topological change (NBCF) and for the atoms with topological change (BCF). The BCF PDF shows drop in the peak height; so to further analyze the data, we show the difference $\Delta g_{0}^{0}(r)$ between the nondeformed (reference) and deformed state in Fig. 5(b). For the atoms without BCF (the top panel), $\Delta g_{0}^{0}(r)$ is very small, and reflects simply the volume change induced by elastic deformation, which is proportional to the first derivative of $g_{0}^{0}(r)$.

$$
\Delta g_{0}^{0}(\mathrm{r})=\varepsilon_{\mathrm{aff}} \frac{1-2 v}{3} \mathrm{r} \frac{\mathrm{d}}{\mathrm{dr}} g_{0}^{0}(\mathrm{r}),
$$


Therefore, the change in the PDF for NBCF atoms can be explained by a volume change

due to the tensile stress which includes expansive pressure. However, $\Delta g_{0}^{0}(r)$ for atoms participating in BCF is much larger, and cannot be fitted using the above formula as seen in the bottom panel of Figure 5(b). As shown in the early study [15] density fluctuation induced by structural rejuvenation results in the changes $g_{0}^{0}(r)$ which are proportional to the second derivative of $g_{0}^{0}(r)$. Here, we also compare $\Delta g_{0}^{0}(r)$ with the second derivative of $g_{0}^{0}(r)$ in Fig. 5b (bottom panel). Interestingly, $\Delta g_{0}^{0}(r)$ stays in between the first and second derivatives of $g_{0}^{0}(r)$, which can be understood as a combination effect of affine elastic deformation and structural rejuvenation because $g_{0}^{0}(r)$ for $\mathrm{BCF}$ atoms is calculated under tensile stress. These results show that the structural rejuvenation can be elucidated in terms of the bond-cutting-formation rearrangements. The irreversible topological changes involved in the BCF process increase the internal energy, and thus the fictive temperature.

\section{Conclusions}

The structural evidence of structural rejuvenation in BMGs induced by strain-rate controlled deformation at high temperature has been obtained via ex-situ high energy Xray diffraction. We observed decrease in the PDF peak height with deformation, which indicated structural disordering. The decrease in the PDF peak height was more pronounced for samples deformed with higher strain rates. The trend was reversed at the highest strain rate close to the onset of inhomogeneous deformation. The experiment was also performed at a constant strain rate but terminating at different final stages. The results showed surge in a plastic strain beyond the overshoot in the steady state regime. 
However, anelastic strain peaked at the maximum stress and did not increase after the stress drop. The changes in the PDF peak height were correlated with the anelastic strain and saturated after the stress drop. Therefore, we conclude that anelastic deformation causes structural rejuvenation. On the other hand plastic deformation during the steady state flow has no effect on the fictive temperature. Importantly most of the rejuvenation processes occur in the anelastic regime before reaching the steady state flow. The anisotropic part of the PDF after strain rate controlled deformation is the same as that after isothermal creep, indicating that the same mechanism is in play for both cases. Because of the correlation between the fictive temperatures and anelastic strain we conclude that local topological structural changes activated by external stress at elevated temperature are responsible for both structural rejuvenation and anelasticity. As shown by molecular dynamics simulation the local topological arrangement consists of bondcutting and formation $(\mathrm{BCF})$ processes. From the practical point of view, BMG material can be rejuvenated at high temperature well before the onset of plastic strain, thus avoiding permanent sample deformation.

\section{Acknowledgments}

This work was supported by the Department of Energy, Office of Sciences, Basic Energy Science, Materials Science and Engineering Division. The authors are grateful to J. S. Langer for useful discussions. 


\section{References}

[1] Schuh CA, Hufnagel TC, Ramamurty U. Acta Mater 2007;55:4067.

[2] Spaepen F. Acta Metall 1977;25:9.

[3] Schroers J, Johnson WL. Phys Rev Lett 2004;93:4.

[4] Yao KF, Ruan F, Yang YQ, Chen N. Appl Phys Lett 2006;88:122106.

[5] Yokoyama Y, Fujita K, Yavari AR, Inoue A. Philos Mag Lett 2009;89:322.

[6] Yokoyama Y, Yamada M, Mori T, Tokunaga H, Sato T, Shima T, Nishijima M, Fujita K, Yamasaki T. Mater Sci Eng A 2014;606:74.

[7] Kumar G, Rector D, Conner RD, Schroers J. Acta Mater 2009;57:3572.

[8] Tong Y, Dmowski W, Yokoyama Y, Wang G, Liaw PK, Egami T. Scripta Mater 2013;69:570.

[9] Murali P, Ramamurty U. Acta Mater 2005;53:1467.

[10] Yokoyama Y, Yamasaki T, Liaw PK, Inoue A. Acta Mater 2008;56:6097.

[11] Park K-W, Lee C-M, Wakeda M, Shibutani Y, Falk ML, Lee J-C. Acta Mater 2008;56:5440.

[12] Yokoyama Y, Yamano K, Fukaura K, Sunada H, Inoue A. Mater Trans 2001;42:623.

[13] Yokoyama Y, Inoue K, Fukaura K. Mater Trans 2002;43:3199.

[14] Zhang Y, Wang WH, Greer AL. Nat Mater 2006;5:857.

[15] Dmowski W, Yokoyama Y, Chuang A, Ren Y, Umemoto M, Tsuchiya K, Inoue A, Egami T. Acta Mater 2010;58:10.

[16] Langer J, Manning M. Phys Rev E 2007;76: 056107.

[17] Faupel F, Frank W, Macht MP, Mehrer H, Naundorf V, Ratzke K, Schober HR, Sharma SK, Teichler H. Rev Mod Phys 2003;75:237.

[18] Ono IK. , O’Hern CS, Durian DJ, Langer SA, A. J. Liu AJ, and Nagel SR. Phys Rev Lett 2002; 89:095703.

[19] Srolovitz D, Egami T, Vitek V. Phys Rev B 1981; 24:6936.

[20] Egami T, Srolovitz D. J Phys F: Met Phys 1982;12:2141.

[21] Yokoyama Y, Inoue K, Fukaura K. Mater Trans 2002;43:2316.

[22] Levashov VA, Egami T, Aga RS, Morris JR. Phys Rev B 2008;78: 064205.

[23] Dmowski W, Iwashita T, Chuang C-P, Almer J, Egami T. Phys Rev Lett 2010;105:205502.

[24] Dmowski W, Egami T. J Mater Res 2007;22:7.

[25] Suzuki Y, Haimovich J, Egami T. Phys Rev B 1987;35:7.

[26] Dmowski W, Egami T. Adv Eng Mater 2008;10:5.

[27] Ott RT, Heggen M, Feuerbacher M, Park ES, Kim DH, Kramer MJ, Besser MF, Sordelet DJ. Acta Mater 2008;56:9.

[28] Tong Y, Dmowski W, Witczak Z, Chuang C-P, Egami T. Acta Mater 2013;61:1204.

[29] Lu J, Ravichandran G, and Johnson WL. Acta Mater 2003; 51: 3429.

[30] Egami T. J Mater Sci 1978;13:2587.

[31] Dmowski W, Fan C, Morrison ML, Liaw PK, Egami T. Mater Sci Eng A 2007;471:125.

[32] Li WD, Bei H, Tong Y, Dmowski W, Gao YF. Appl Phys Lett 2013;103: 171910.

[33] Harmon JS, Demetriou MD, Johnson WL, Samwer K. Phys Rev Lett 2007;99:4.

[34] Argon AS. Acta Metall 1983;31:499. 
Figures
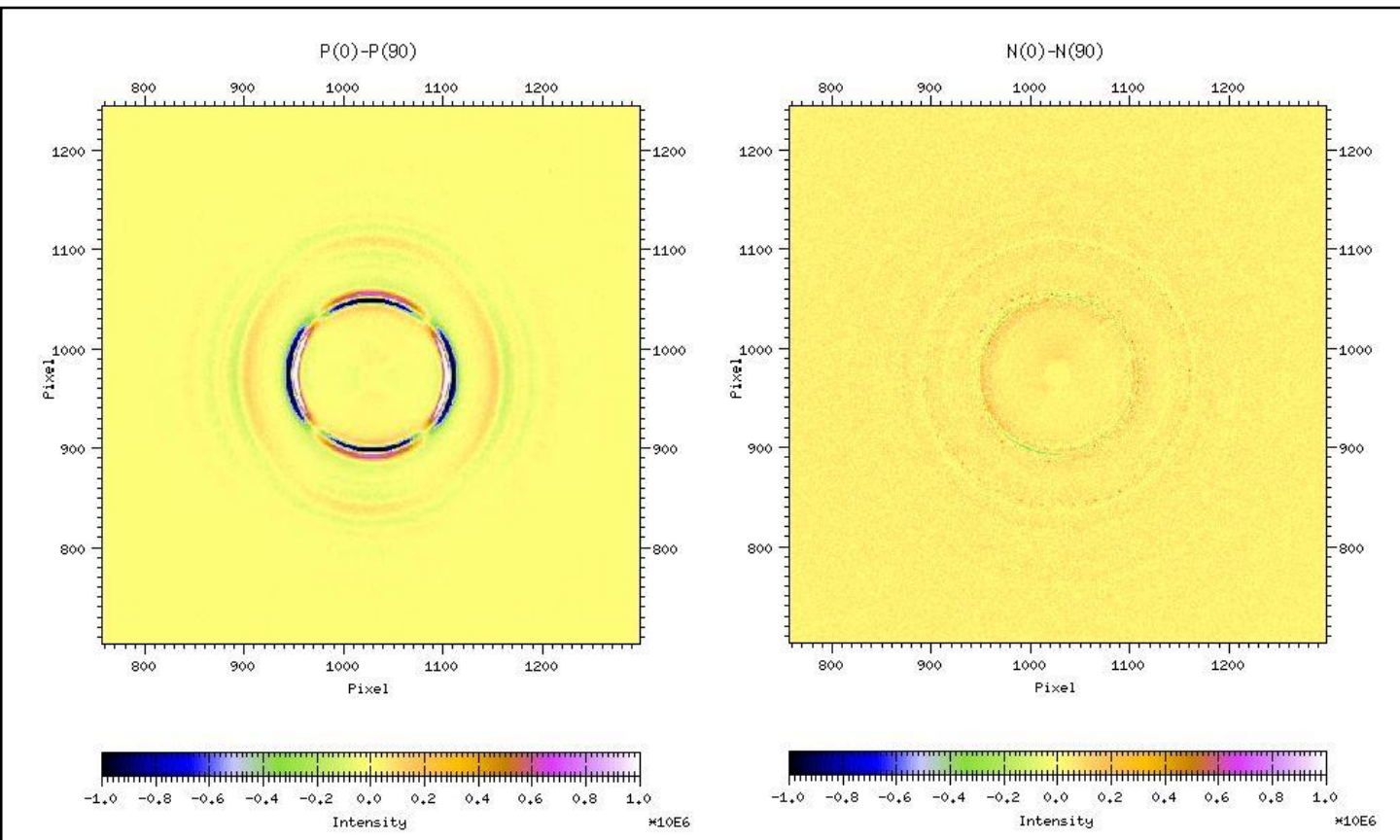

Figure 1. The difference of 2-D diffraction patterns between $0^{\circ}$ and $90^{\circ}$ orientations for planes parallel and normal to stress.
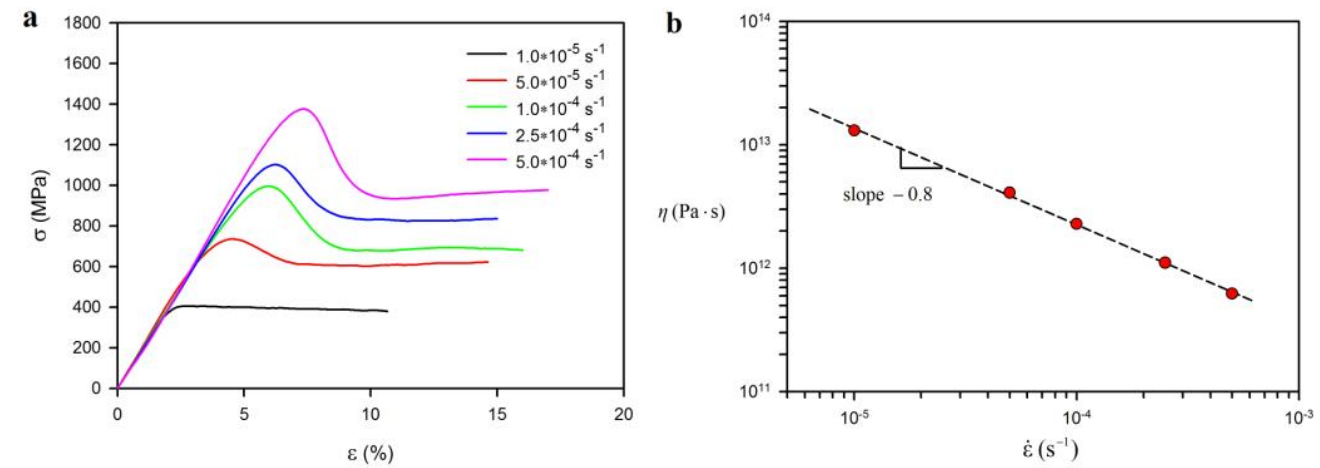

Figure 2.(a) Stress-strain curves for samples deformed with different strain rate. From these curves flow stress $\left(\sigma_{f}\right)$ is obtained for each strain rate and viscosity is calculated by using equation: $\sigma_{f}=3 \eta \dot{\varepsilon}$. (b) Viscosity-strain rate relationship. 


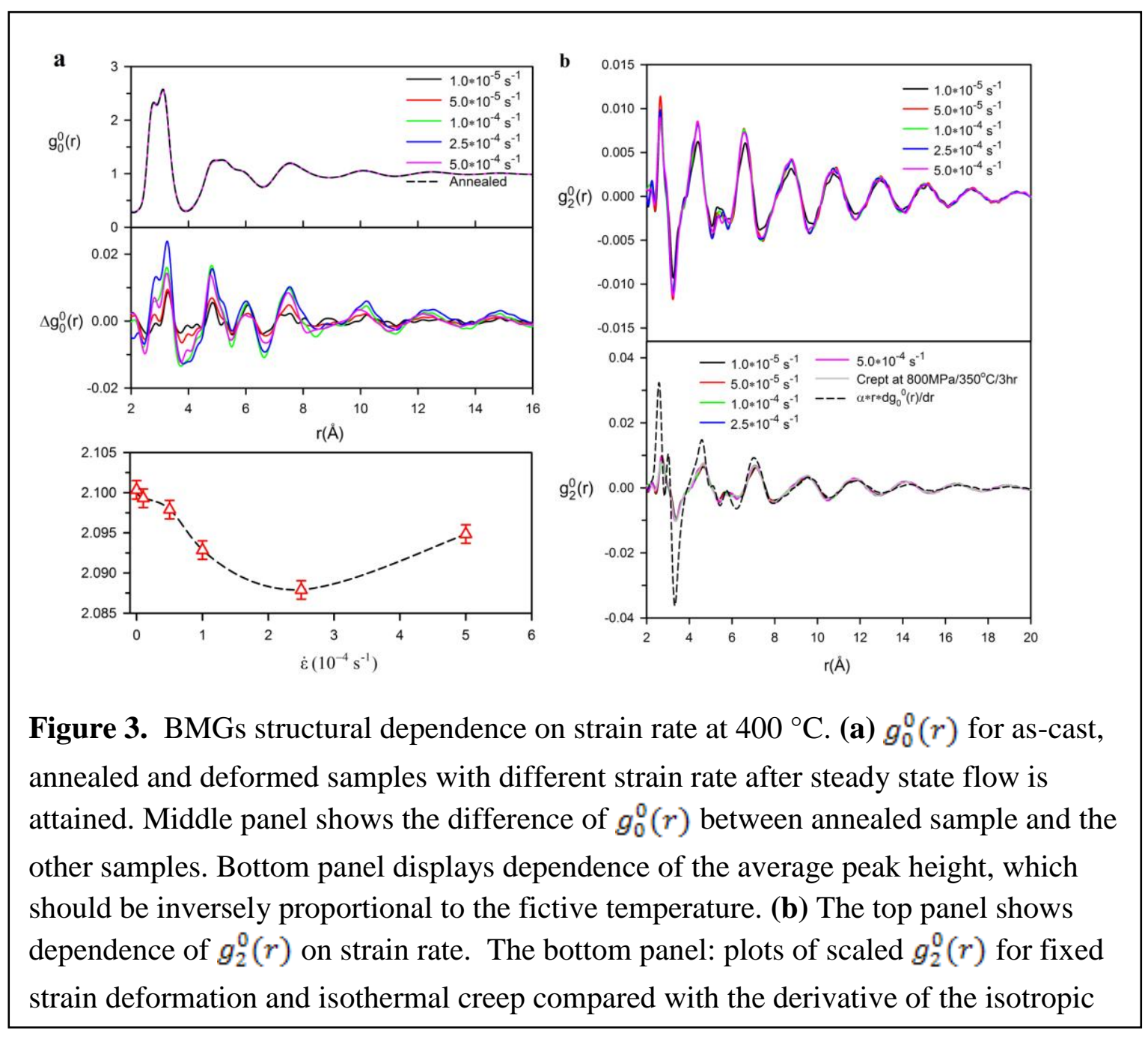




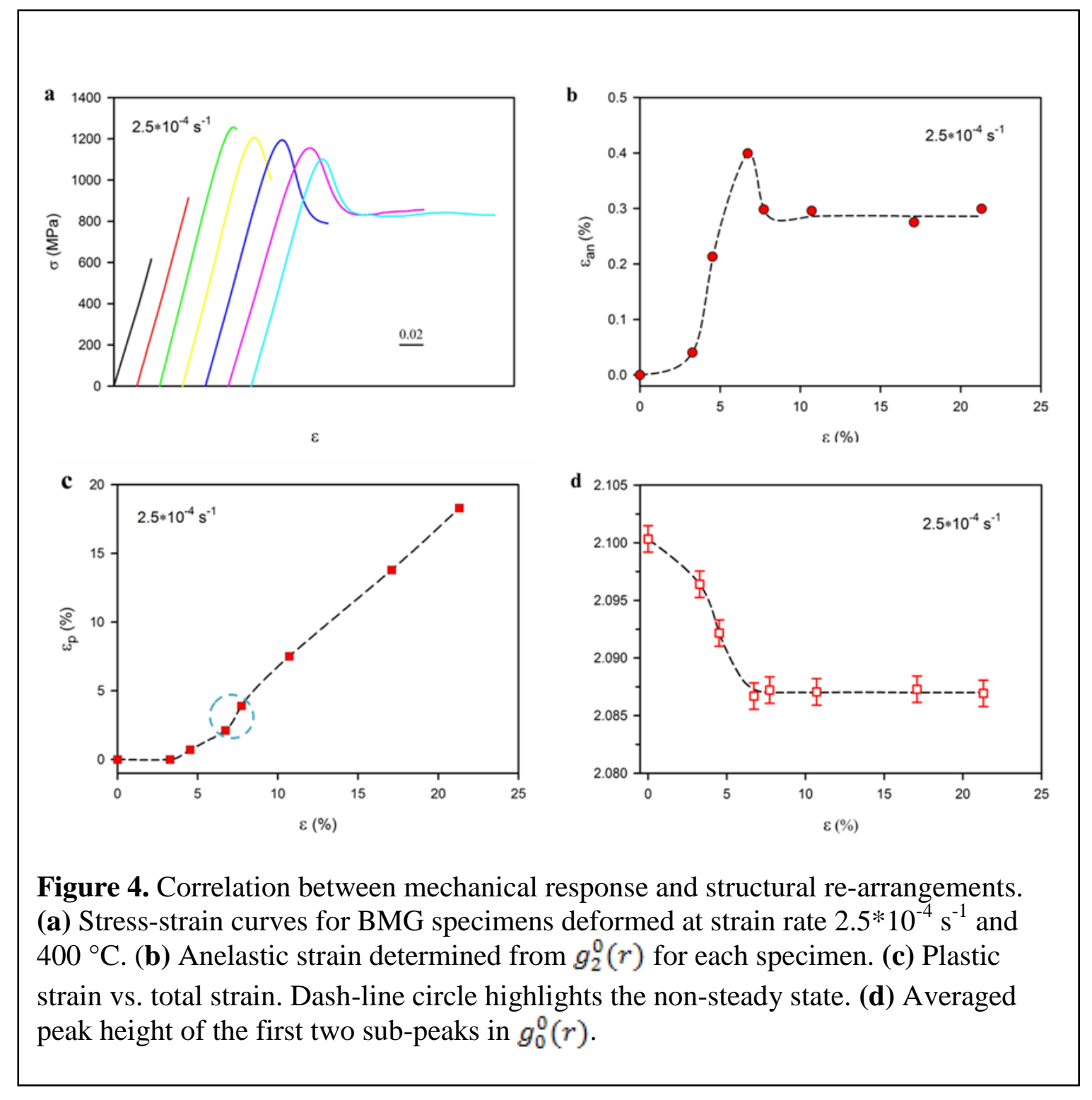



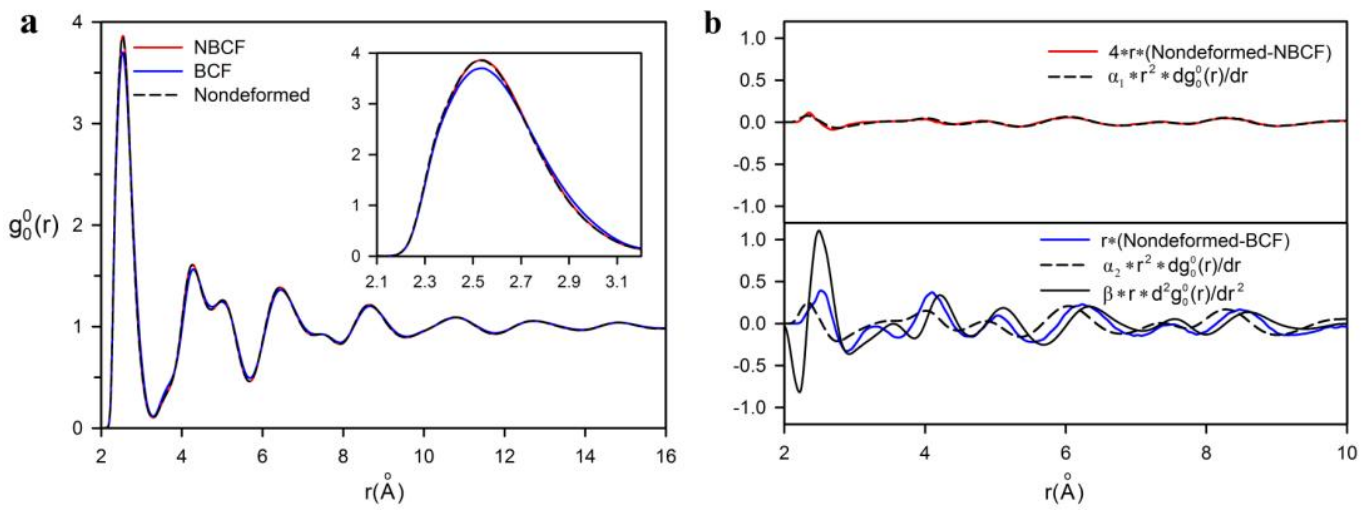

Figure 5. The influence of topological change on $g_{0}^{0}(r)$. (a) $g_{0}^{0}(r)$ calculated for all simulated atoms without external stress (no deformation), atoms without topological change (NBCF) under stress, and atoms experiencing BCF under stress, respectively. First peak of $g_{0}^{0}(r)$ is highlighted in insert. (b) Top panel: difference in $g_{0}^{0}(r)$ between reference PDF and for atoms without topological change (NBCF); bottom panel: difference in $g_{0}^{0}(r)$ between reference PDF and for atoms with topological change (BCF) compared with first and second derivative of $g_{0}^{0}(r)$. Multiplying by $r$ is to strengthen oscillation in high $r$ range. 